\title{
CHAPTER 3B.
}

\section{Surveys and Observations: \\ Local Universe}




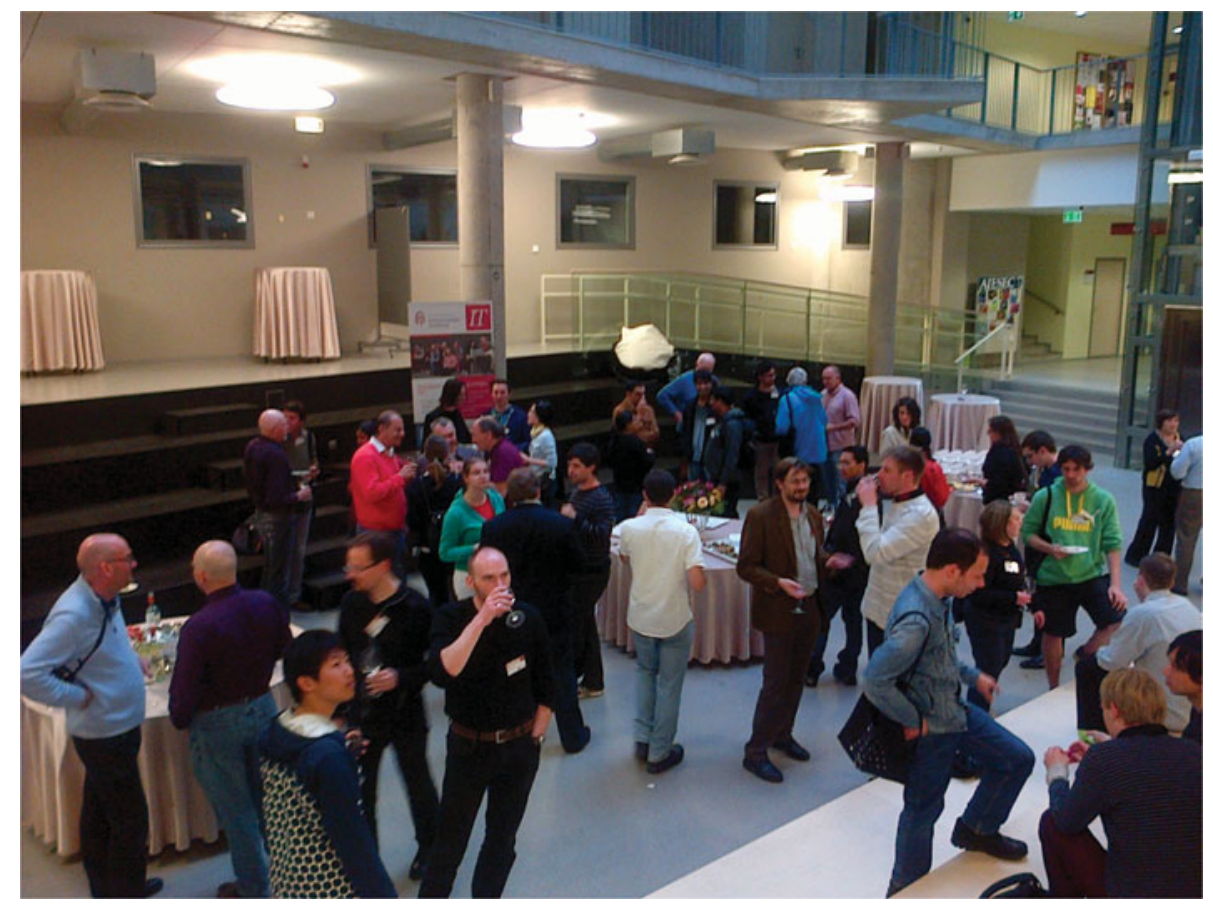

IAU308 symposium gathers for the official opening reception. Photo courtesy: Peter Coles

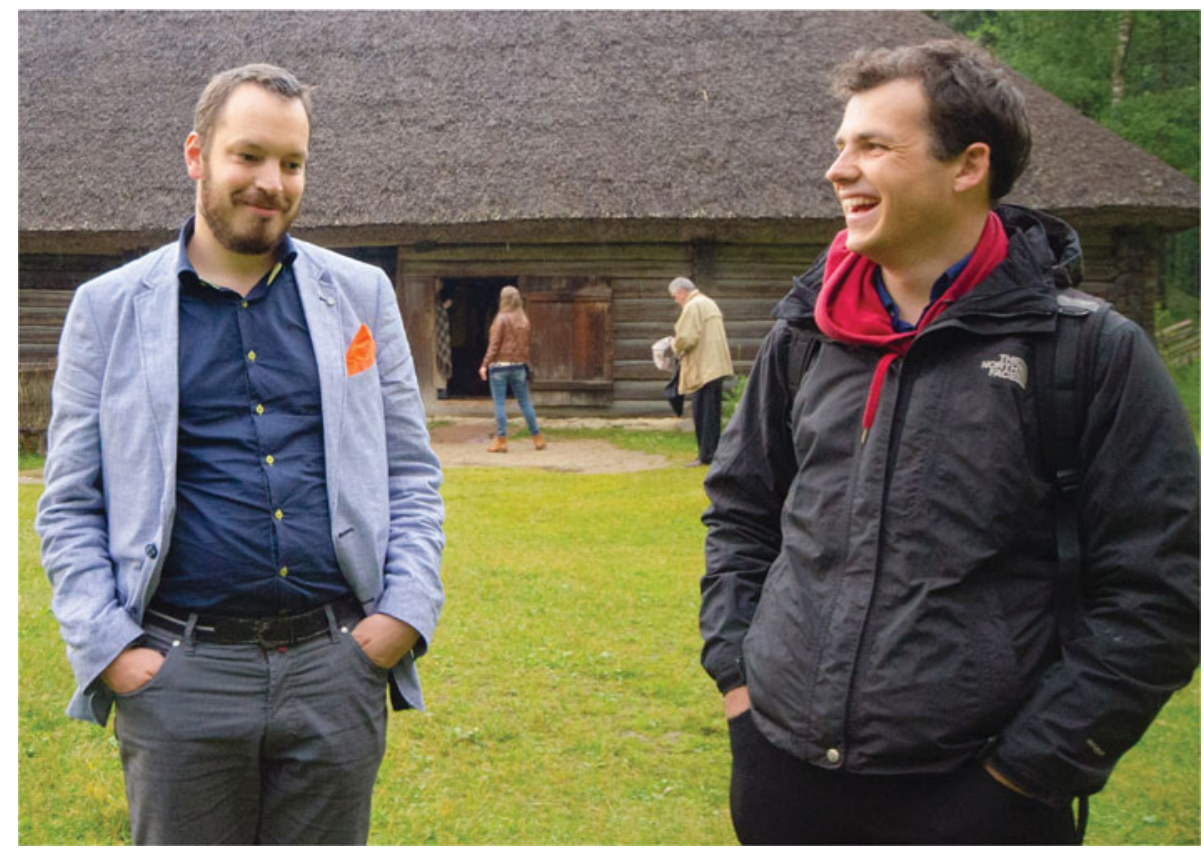

Caustics in the Local Universe:

Johan Hidding \& Job Feldbrugge in front of an Estonian farmhouse Photo courtesy: Steven Rieder 


\title{
Dynamics of galaxy structures in the Local Volume
}

\author{
I. D. Karachentsev \\ Special Astrophysical Observatory, \\ Russian Academy of Sciences N.Arkhyz, KChR, 369167, Russia \\ email:ikar@sao.ru
}

\begin{abstract}
I consider a sample of 'Updated Nearby Galaxy Catalog' that contains eight hundred objects within $11 \mathrm{Mpc}$. Environment of each galaxy is characterized by a tidal index $\Theta_{1}$ depending on separation and mass of the galaxy Main Disturber $(=\mathrm{MD})$. The UNGC galaxies with a common MD are ascribed to its 'suite' and ranked according to their $\Theta_{1}$. Fifteen the most populated suites contain more than half of the UNGC sample. The fraction of MDs among the brightest galaxies is almost $100 \%$ and drops to $50 \%$ at $M_{B}=-18$ mag. The observational properties of galaxies accumulated in UNGC are used to derive orbital masses of giant galaxies via motions of their satellites. The average orbital-to-stellar mass ratio for them is $M_{\text {orb }} / M_{*} \simeq 30$, corresponding to the mean local density of matter $\Omega_{m} \simeq 0.09$, i.e $1 / 3$ of the global cosmic one. The dark-to-stellar mass ratio for the Milky Way and M31 is typical for other neighboring giant galaxies.
\end{abstract}

Keywords. cosmology: observations - dark matter - galaxies: groups: general

\section{Introduction and the data sample}

As observational data show, the bulk of galaxies inhabit the groups, like our Local Group. Due to their cosmic abundance, the galaxy groups make a main contribution to the average density of matter in the universe. Most of the groups are concentrated in the filaments and sheets, forming a large-scale 'cosmic web' (Zeldovich 1970, Shandarin et al. 2004, Einasto et al. 2011). Until recently, the scarcity of data on the distances of even the closest galaxies was the major obstacle in the development of observational cosmology in the Local universe.

The recently published Updated Nearby Galaxy Catalog (= UNGC, Karachentsev et al., 2013) contains a summary of data on radial velocities, distances and other observable parameters of about 800 galaxies located within a $11 \mathrm{Mpc}$ radius around us. More than 300 galaxies of this sample have accurate distance measurements with a (510)\% accuracy obtained by the Tip of the Red Giant Branch from observations with the Hubble Space Telescope. Due to the proximity of the UNGC- objects, the kinematic data density in the catalog proves to be one order higher than in the sample of Sloan Digital Sky Survey (Abazajian et al. 2009). This circumstance, and the presence of individual distance measurements in many UNGC galaxies allows us to investigate the structure of nearby groups and their vicinities with unprecedented detail.

\section{Neighboring giants and their suites}

Possessing the data on the distances and luminosities of eight hundred galaxies of the Local Volume, Karachentsev et al. (2013) have determined for each galaxy its tidal index

$$
\Theta_{1}=\max \left[\log \left(M_{n}^{*} / D_{n}^{3}\right)\right]+C, n=1,2, \ldots N,
$$




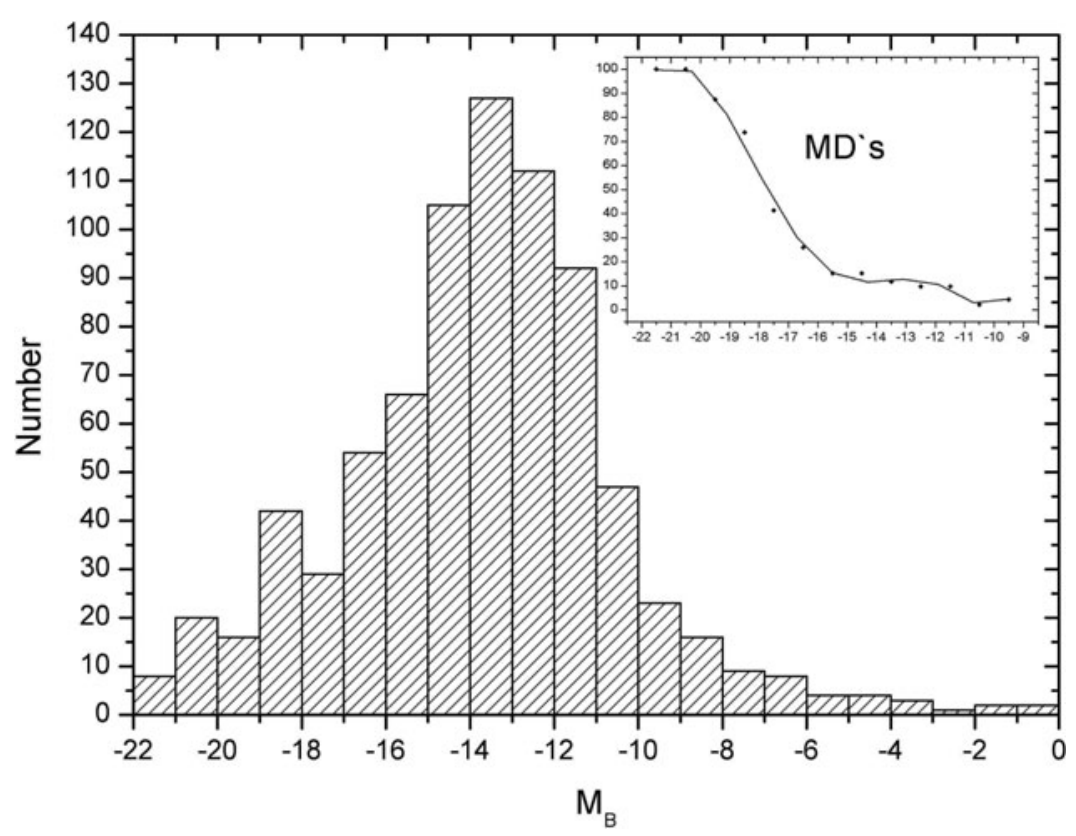

Figure 1. Distribution of 795 galaxies in a sphere of $11 \mathrm{Mpc}$ radius around the Milky Way on absolute $B$-magnitudes, corrected for the internal and external extinction. The inset shows what percentage of these galaxies in each bin act as the Main Disturber.

where $M^{*}$ is the stellar mass of the neighboring galaxy, and $D_{n}$ is its spatial separation from the considered galaxy. The stellar mass of the galaxy was assumed to be equal to its K-band luminosity at $M / L_{K}=1 \times M / L$. Ranking the surrounding galaxies by the magnitude of their tidal force, $F_{n} \sim M^{*} / D^{3}$, allowed to find the most influential neighbor, called the Main Disturber $(=\mathrm{MD})$. Here the ratio of the total mass of the galaxy to its stellar mass was considered to be constant regardless of the luminosity and morphology of galaxies. The constant $C=-10.96$ was chosen so that the galaxy with $\Theta_{1}=0$ was located at the zero velocity sphere relative to its MD. In other words, the galaxy with $\Theta_{1} \geqslant 0$ was regarded as gravitationally related to its MD as their crossing time was shorter than the age of the universe.

The galaxies which have a common MD can be combined in a certain association, or a MD suite. At that, an aggregate of suite members with positive values is quite consistent with the notion of a physically bound group of galaxies. The most massive MDs possess the most populous suites. The total number of companions around 15 most massive galaxies makes up about a half of the total population of the Local Volume.

Fig. 1 represents the distribution of galaxies of the Local Volume by the absolute Bband magnitude. The inset picture shows what fraction of the MDs as function of the absolute magnitude. The relative number of MDs among the brightest galaxies is close to $100 \%$. The fraction of MDs decreases towards the low-luminosity galaxies, dropping below $50 \%$ at $M_{B}-18 \mathrm{mag}$.

The distribution of 351 companions by the radial velocity difference and projection separation relative to their main galaxies is presented in three panels of Fig.2. The upper panel of the figure shows the $\left\{\Delta_{V}, R_{p}\right\}$ diagram for 31 companions of the Milky Way $=\mathrm{MW}$ (squares) and 39 members of the M $31=$ Andromeda suite (diamonds). The companions of massive galaxies with the positive tidal index, considered to be physical, are represented by closed symbols, while the members of the suites with $\Theta_{1}=(0,-0.5)$ 


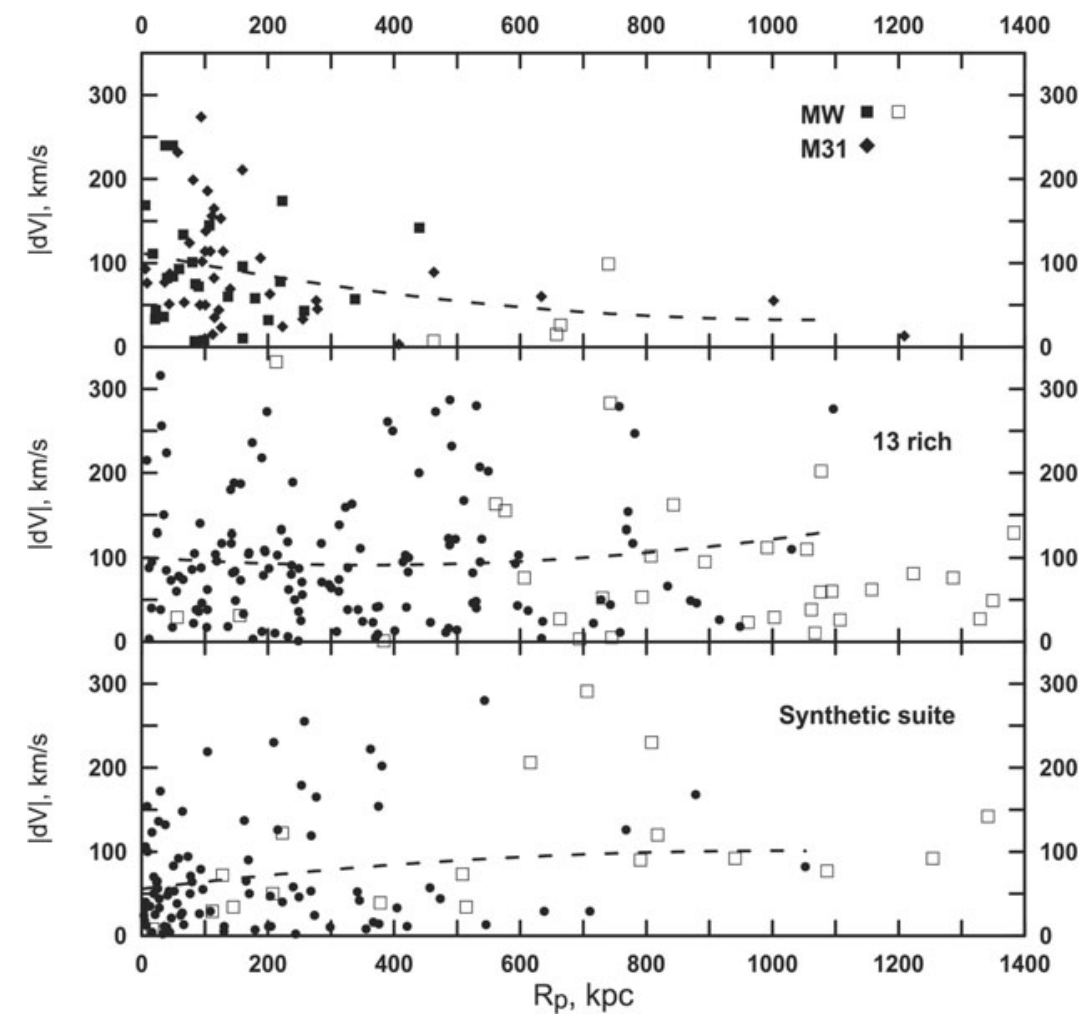

Figure 2. Line-of-sight velocity of the suite members relative to the main galaxy as a function of their projected linear separation.

are shown by the open symbols. The extension of the companion sample by the objects with slightly negative values of was done not to miss some possible physical members of the group, in which the distances are as yet measured with low accuracy. The objects in this boundary category may appear to be both the real companions of main galaxies or belong to the population of general field.

The middle panel of Fig.2 shows the $\left\{\Delta V, R_{p}\right\}$ distribution for 174 members of rich suites around 13 other massive nearby galaxies. Prospective physical companions $(\mathrm{N}=$ 142) are also marked here by solid symbols. In addition to 15 rich suites, the Local Volume comprises a lot of small suites, where the radial velocities are measured in one or several presumed companions. We have combined these small suites in a composite suite. The $\left\{\Delta V, R_{p}\right\}$ diagram for 107 companions uniting small suites is represented on the lower panel of Fig. 2. The dashed lines in all the three panels show quadratic regressions of the velocity difference on the projection separation of companions.

\section{Orbital and stellar masses}

If the group is dominated by a massive galaxy, surrounded by a set of test particles with random orientation of their orbits, one can use the mass estimate (Karachentsev,2005):

$$
M_{\text {orb }}=(16 / \pi) \times G^{-1} \times\left\langle\Delta V_{12}^{2} \times R_{p 12}\right\rangle,
$$

where $G$ is the gravitational constant, $\Delta V_{12}$ and $R_{p 12}$ are the velocity difference and the projected separation of companions relative to the main galaxy. The prevailing orbit 
Table 1. Basic properties of the nearby suites.

\begin{tabular}{|l|c|c|c|c|c|c|}
\hline MD & $N$ & $N_{v}$ & $\begin{array}{c}\left\langle R_{p}\right\rangle \\
\mathrm{kpc}\end{array}$ & $\begin{array}{c}\langle\Delta V| \mathrm{km} \mathrm{s}^{-1} \\
\mathrm{~km}\end{array}$ & $\begin{array}{c}M_{M D}^{*} \operatorname{dex}_{(10) M_{\odot}} \\
\text { dex }(12) M_{\odot}\end{array}$ \\
\hline MW & 38 & 27 & 121 & 90 & 3.5 & $1.44 \pm 0.46$ \\
M31 & 42 & 39 & 198 & 93 & 5.4 & $1.76 \pm 0.33$ \\
M81 & 53 & 26 & 219 & 116 & 8.5 & $4.89 \pm 1.41$ \\
N5128 & 37 & 15 & 343 & 110 & 8.1 & $6.71 \pm 2.09$ \\
N4594 & 32 & 6 & 577 & 153 & 20.0 & $28.47 \pm 17.80$ \\
N3368 & 31 & 20 & 408 & 150 & 6.8 & $17.00 \pm 4.30$ \\
N4258 & 31 & 11 & 316 & 96 & 8.7 & $3.16 \pm 1.01$ \\
N4736 & 31 & 14 & 515 & 50 & 4.1 & $2.67 \pm 0.90$ \\
N5236 & 28 & 10 & 294 & 57 & 7.2 & $1.06 \pm 0.28$ \\
N253 & 25 & 7 & 500 & 51 & 11.0 & $1.51 \pm 0.59$ \\
N3115 & 12 & 6 & 215 & 82 & 8.9 & $3.43 \pm 2.00$ \\
M101 & 11 & 6 & 167 & 76 & 7.1 & $1.47 \pm 0.67$ \\
IC342 & 10 & 8 & 321 & 66 & 4.0 & $1.810 \pm .82$ \\
N3627 & 8 & 7 & 254 & 69 & 10.2 & $1.45 \pm 0.39$ \\
N6946 & 8 & 6 & 163 & 60 & 5.8 & $0.66 \pm 0.34$ \\
\hline
\end{tabular}

The columns contain: (1) name of the suite/group by its main galaxy, (2) the total number of satellites, (3) the number of physical companions with measured radial velocities, (4) the average projection separation of the companions from the MD, (5) the mean absolute value of the radial velocity difference of the companions relative to the $\mathrm{MD},(6)$ the main galaxy stellar mass, (7) the value of orbital mass of the group.

eccentricity is assumed to be $1 / \sqrt{2}$. The basic characteristics of the considered suites are presented in Table 1.

\section{The Milky Way and Andromeda suites as compared with others.}

Modeling the structure and kinematics of galaxy groups within the $\Lambda$ CDM paradigm, many authors (Libeskind et al. 2010, Knebe et al. 2011) choose the Local Group to make a comparison with the observational data. Previously Karachentsev et al. (2014) noted that judging on some morphological features the groups of galaxies around the MW and M31 are not quite typical.

Six histograms of Fig. 3 represent the distributions of 15 most populated suites in the Local Volume based on their global parameters. The groups of galaxies around the MW and Andromeda are marked with ' $\mathrm{M}$ ' and 'A', respectively. The histogram data show that based on their stellar masses, and orbital masses, both MW and M 31 do not get in the top ten most massive galaxies of the Local Volume, whereas based on the orbital-to-stellar mass ratio $\sim 30$ both groups are not significantly different from the rest.

\section{Masses derived from Hubble flows around the nearby groups.}

A high density of observational data on the radial velocities and distances of galaxies in the Local Volume gives an opportunity to determine the masses of nearby groups not only by the virial motions, but also by perturbations of the Hubble flow around them. This idea was proposed by Lynden-Bell (1981), and is based on the measurement of the radius of the zero velocity sphere, $R_{0}$, which separates a group from the surrounding volume that expands. In the standard cosmological model with the parameters $H_{0}=73$ $\mathrm{km} \mathrm{s}^{-1} \mathrm{Mpc}^{-1}$ and $\Omega_{m}=0.24$ the total mass of a spherical overdensity is expressed as

$$
M_{T} / M_{\odot}=2.12 \times 10^{12} \times\left(R_{0} / \mathrm{Mpc}\right)^{3} .
$$

An important circumstance here is that the estimate of the total mass of a group corresponds to the scale of $R_{0}$, which is $\sim 3.7$ times larger than its virial radius. The analysis of observational data on radial velocities and separations of galaxies in the 


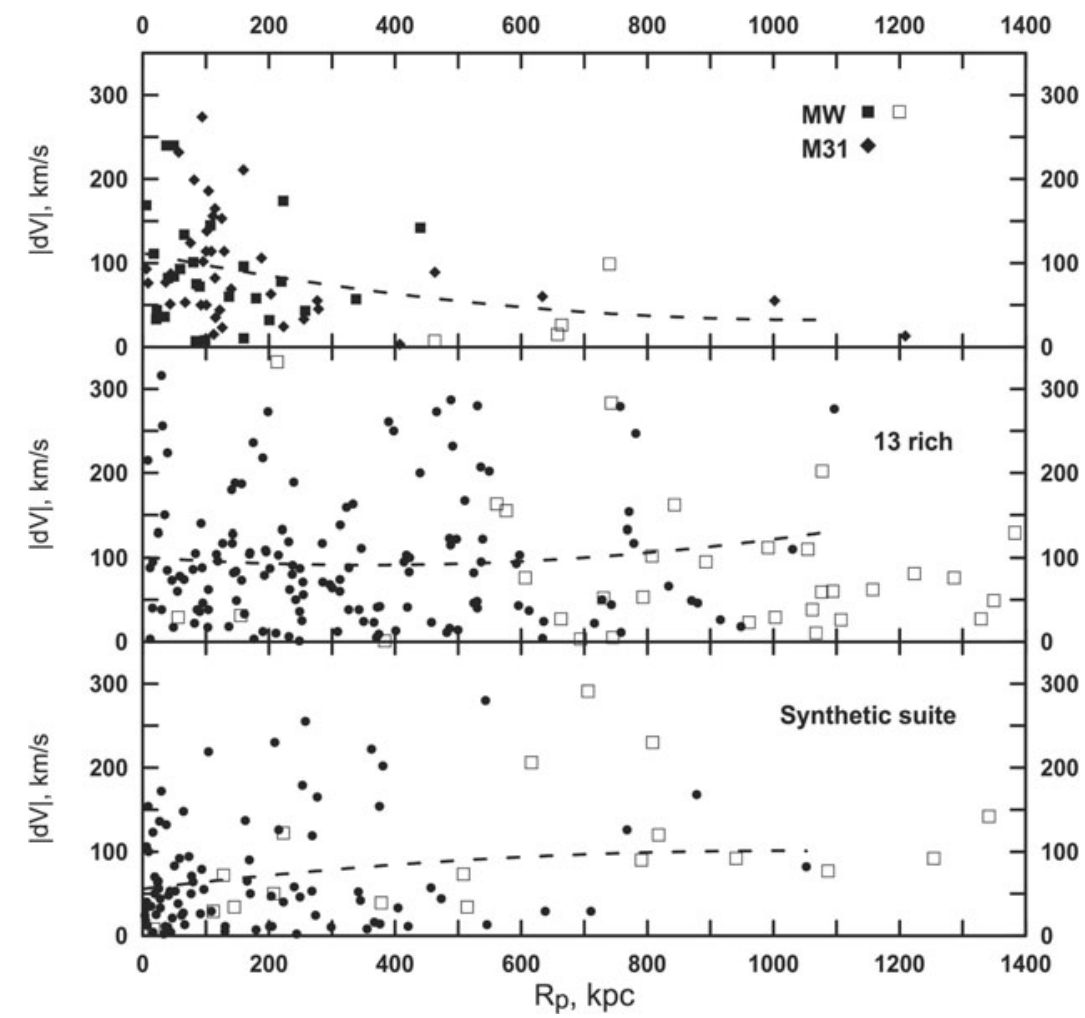

Figure 3. The distributions of the 15 richest nearby suites according to: a) the mean projected separation of physical companions, b) radial velocity dispersion, c) logarithm of stellar mass of the main galaxy, d) logarithm of the orbital mass, e) the mean orbital-to-stellar mass ratio, $f$ ) the mean crossing time for the components in units of the global cosmic time $T_{0}$. The Milky Way suite and the Andromeda (M31) suite are depicted by ' $\mathrm{M}$ ' and 'A', respectively.

Table 2. Total masses of nearby groups via internal and external motions.

\begin{tabular}{l|l|l|l|l|}
\hline Group & $\log \left(M_{\text {orb }}\right)$ & $R_{0},(\mathrm{Mpc})$ & $\log \left(M_{\text {tot }}\right)$ & $\log \left(M_{T} / M_{\text {orb }}\right) \mid$ \\
\hline MW+M31 & $12.52 \pm 0.08$ & $0.98 \pm 0.03$ & $12.30 \pm 0.05$ & $-0.22 \pm 0.09$ \\
IC342 & $12.26 \pm 0.21$ & $0.90 \pm 0.10$ & $12.19 \pm 0.14$ & $-0.07 \pm 0.25$ \\
M81 & $12.69 \pm 0.13$ & $1.05 \pm 0.07$ & $12.39 \pm 0.09$ & $-0.30 \pm 0.16$ \\
N5128+N5236 & $12.89 \pm 0.14$ & $1.26 \pm 0.15$ & $12.63 \pm 0.15$ & $-0.23 \pm 0.21$ \\
N253 & $12.18 \pm 0.18$ & $0.70 \pm 0.10$ & $11.86 \pm 0.18$ & $-0.32 \pm 0.25$ \\
N4736 & $12.43 \pm 0.15$ & $1.04 \pm 0.20$ & $12.38 \pm 0.24$ & $-0.05 \pm 0.28$ \\
\hline
\end{tabular}

vicinity of the Local Group and other nearby groups was done by different authors. A summary for six groups is presented in Table 2. The references to derived masses are given in Karachentsev \& Kudrya (2014).

In general, the estimates of mass by two independent methods agree with each other quite well. However, a moderate systematic difference of mass estimates in favour of the orbital masses is noteworthy. For six groups the mean log-difference amounts to $-0.20 \pm 0.05$. This paradoxical result lying in the fact that the estimates of the total mass of the groups on the scale of $R_{0} \sim 3.7 R_{v}$ are lower than the orbital mass estimates on the scale of the virial radius $R_{v}$ can have a simple interpretation. Chernin et al. (2013) noted that the estimate of the total mass of a group includes two components: $M_{T}=M_{M}+M_{D E}$, where $M_{M}$ is the mass of dark and baryonic matter, and $M_{D E}=$ 
$(8 \pi / 3) \times \rho_{D E} \times R^{3}$ is the mass, negative in magnitude, determined by the dark energy with the density of $\rho_{D E}$. On the scale of $R_{v}$ the contribution of this component in the group mass is small, not exceeding $1 \%$. But in the sphere of $R_{0}$ radius, the role of this 'mass defect' becomes significant, reaching about $40 \%$. A correction to the total mass by a factor of 1.4 can almost completely eliminate the observed discrepancy between the group mass estimates at different scales.

In turn, such an agreement of mass estimates by the internal and external motions after the correction for the dark energy component can be interpreted as another empirical evidence for the existence of the dark energy itself appearing in the dynamics of nearby groups.

\section{Concluding remarks.}

The high-density data on the distances and radial velocities of $\sim 800$ most nearby galaxies from the UNGC catalog provides an unique opportunity to investigate the distribution of light and dark matter in the Local Volume in outstanding detail.

For the mass of dark halo around the MW and around M 31, we have obtained the values of $(1.35 \pm 0.47)$ and $(1.76 \pm 0.33)$ in the units of $10^{12} M_{\odot}$, respectively. Within the Local Volume, there are 15 rich groups containing more than $50 \%$ of the total Local Volume population. The typical ratio of orbital-to-stellar mass for them is $\sim 30$. This quantity is confirmed by independent estimates of total masses derived from Hubble flows around the groups. The ratio of $M_{o r b} / M^{*} \simeq 30$ corresponds to the mean local density of matter $\Omega_{m} \simeq 0.09$, which is only $1 / 3$ of the global cosmic density of matter.

\section{Acknowledgements}

My co-laborators in this work are Yuri Kudrya, Dmitry Makarov and Elena Kaisina. This work was supported by the grant of Russian Foundation for Basic Research 13-0290407 Ukr-f-a and the grant of the Ukraine F53.2/15.

\section{References}

Abazajian, K. N., Adelman-McCarthy, J. K. \& Agüeros, M. A. et al. 2009, ApJS 182, 543

Chernin, A. D., Bisnovatyi-Kogan, G. S., Teerikorpi, P., Valtonen, M. J, Byrd, G. G. \& Merafina, M. 2013, A\& $A 553,101$

Einasto, J., Hütsi, G., Saar, E., Suhhonenko, I., Liivamgi, L. J, Einasto, M., Müller, V.,

Starobinsky, A. A., Tago, E. \& Tempel, E. 2011, A\&A 531A, 75

Karachentsev, I. D. \& Kudrya, Y.N. 2014, AJ 148, 50

Karachentsev, I. D., Kaisina, E. I. \& Makarov, D.I. 2014, AJ 147, 13

Karachentsev, I. D., Makarov, D. \& Kaisina, E. 2013, $A J$ 145, 101 (=UNGC)

Karachentsev I. D. 2005, AJ 129, 178

Knebe, A., Libeskind, N. I., Doumler, T., Yepes, G., Gottlöber, S. \& Hoffman, Y. 2011, MNRAS $417 \mathrm{~L}, 56$

Libeskind N. I., Yepes G., Knebe A., Gottlöber, S., Hoffman, Y. \& Knollmann, S.R. 2010, MNRAS 401, 1889

Lynden-Bell, D. 1981, Observatory 101, 111

Shandarin, S. F., Sheth, J. V. \& Sahni V. 2004, MNRAS 353, 162

Zeldovich, Ya. B., 1970, A\&A 5, 84 